\title{
ALIANZAS, PODER Y POLÍTICA SOCIAL EN ARGENTINA ${ }^{1}$
}

\section{ALLIANCES, POWER AND SOCIAL POLICY IN ARGENTINA}

\author{
Carl Friedrich Bossert*
}

RESUMEN

\begin{abstract}
A partir de la crisis a comienzos del nuevo siglo se ha ampliado significativamente la protección social para los estratos de bajos ingresos en Argentina. El presente artículo analiza las dinámicas políticas, las alianzas sociales, las relaciones de poder y las estructuras institucionales que conforman el trasfondo político del desarrollo reciente de la política social en Argentina. Se concluye que la transformación de la política social está relacionada con el fortalecimiento de los sectores marginalizados de las clases populares como actores políticos y su incorporación a la alianza gubernamental de centro-izquierda.
\end{abstract} PALABRAS CLAVE: ARGENTINA * POLÍTICA SOCIAL * REDISTRIBUCIÓN DEL INGRESO * PODER
POLÍTICO * TEORÍA POLIITICA

\section{ABSTRACT}

Social protection for low income earners in Argentina has been significantly expanded since the crisis at the turn of the century. The article analyzes the political dynamics, social alliances, power balances and institutional structures that underlie the recent development of social policy in Argentina. It concludes that the transformation of social policy was linked to the strengthening of the lower popular classes as political actors and their incorporation into a centre-left governmental alliance.

\section{KEYWORDS: ARGENTINA * SOCIAL POLICY * INCOME REDISTRIBUTION * POWER RESOURCES * POLITICAL THEORY}

Entre mayo y julio de 2014, el autor estuvo asociado al Programa Nuevas Formas de Acumulación, Distribución y Desigualdad (PADD) del Instituto de Investigaciones Sociales (IIS) de la Universidad de Costa Rica. Actualmente está terminando su tesis doctoral sobre la economía política de la protección social para personas de bajos ingresos en América Latina. La recolección de datos y tareas de investigación para este artículo fueron llevadas a cabo a lo largo de numerosas estadías de investigación en Argentina entre 2010 y 2014. El autor agradece a la Fundación Hans-Böckler, al Servicio Alemán de Intercambio Académico (DAAD, por sus siglas en alemán) y a la Universidad de Viena, que proporcionaron el financiamiento para dichas estadías de investigación. El autor agradece además a sus colegas del GSPG y del PADD y a un revisor anónimo que han contribuido a este artículo con comentarios y críticas. Koen Voorend se tomó el tiempo de revisar el documento completo y hacer comentarios sumamente valiosos. Ezequiel Luis Bistoletti merece un especial agradecimiento, dado que muchas de las ideas en este artículo surgieron a partir de un trabajo conjunto realizado para una ponencia sobre políticas sociales en Venezuela y Argentina. 


\section{INTRODUCCIÓN}

Durante los últimos quince años, la política social ha experimentado cambios sustanciales en varios países de América Latina. Por un lado, se puede observar la expansión de una variedad de programas de asistencia social $y$ de desarrollo social, como los programas de vivienda, educación, atención médica y transferencias monetarias condicionadas (Comisión Económica para América Latina y el CaribeCEPAL 2013 y 2014). Por otro lado, algunos países han reformado sus seguros sociales con el fin de mejorar el acceso de los grupos de bajos ingresos (Arza 2009; Becker y Mesa-Lago 2013). Mientras que los Estados de bienestar de América Latina han sido comúnmente descritos como "truncados" (de Ferranti 2004 y Fiszbein 2005), es decir, que cubren solo algunos segmentos relativamente pequeños y privilegiados de la población, las recientes reformas han ampliado la cantidad de recursos destinados a los sectores más pobres de la sociedad. De este modo, han contribuido significativamente a la disminución de la pobreza y la desigualdad y han mejorado las condiciones de vida de millones de latinoamericanos (CEPAL 2013; Barrientos 2012; Lustig, Lopez-Calva y Ortiz-Juarez 2012).

Desde el inicio de este proceso, una amplia gama de estudios han analizado el aspecto programático del cambio. Sin embargo, pocos son los estudios que analizan las dinámicas políticas que dieron origen a estos cambios: ¿cómo se explica la expansión de la política social dirigida hacia los sectores marginalizados de las clases populares?2 ${ }^{2}$

Este artículo reconstruye los procesos políticos que condujeron a la expansión de las políticas sociales dirigidas a los sectores económicamente marginados de Argentina con el fin de mejorar la comprensión de las causas y de los mecanismos políticos subyacentes.

$2 \quad$ La noción "sectores marginalizados de las clases populares" se refiere a los sectores de bajos ingresos que por lo general no han sido representados por los sindicatos en Argentina y que han carecido de acceso a los sistemas de seguro social, a los que han logrado acceder otros sectores de las clases populares.
Argentina es un caso emblemático porque muestra una expansión particularmente fuerte de la protección social a los grupos de bajos ingresos. Además, en dicho país el contraste con las reformas sociales altamente regresivas de la década anterior es más fuerte que en cualquier otro país de América Latina. Por lo tanto, constituye un caso especialmente propicio para indagar las causas y los mecanismos políticos de este cambio.

El análisis muestra que la expansión de las políticas redistributivas dirigidas a los sectores marginalizados de las clases populares está vinculado con un proceso de incorporación de estos sectores al corazón de la alianza gubernamental del "kirchnerismo". A su vez, esta recomposición de la alianza gubernamental, ha de entenderse como un proceso que involucró conflictos $y$ negociaciones entre varios actores, algunos de los cuales perdieron influencia sobre las políticas estatales mientras otros la ganaron. En estos conflictos y negociaciones, la política social constituyó un elemento clave que permitió la construcción y consolidación de la nueva alianza al proveer importantes beneficios para los actores incorporados. Para comprender esta recomposición de la alianza gubernamental es necesario tomar en cuenta el cambio de la distribución de recursos de poder hacia actores progresistas, incluyendo el fortalecimiento de los movimientos de los trabajadores desempleados. Paralelamente, se dio un creciente descrédito del pensamiento neoliberal, del sector financiero $y$ de los organismos financieros internacionales en la opinión pública, lo cual debilitó a un sector de la élite particularmente opuesto a las políticas redistributivas.

\section{MARCO ANALÍTICO Y METODOLÓGICO}

El marco analítico se basa en la teoría de recursos de poder (Esping-Andersen 1990; Korpi 1983; Huber y Stephens 2001) y en los enfoques teóricos que han relacionado las tendencias en las políticas redistributivas con la conformación de variadas alianzas gubernamentales (Collier y Collier 2002; Haggard y Kaufman 2008; Martínez y Voorend 2009; O'Donnell 1977). 
La teoría de los recursos de poder propone un marco conceptual para el análisis de relaciones de poder. Este marco ha sido extensamente utilizado para analizar patrones de conflicto y cooperación entre actores, así como, resultados de las luchas de intereses que se dan en el ámbito de la política social. Originalmente, este enfoque fue desarrollado para captar empíricamente las relaciones de poder entre clases sociales (Esping-Andersen 1990 y Korpi 1983), pero luego se extendió a otras relaciones de poder (Hobson y Lindholm 1997; Huber y Stephens 2001), particularmente a las relacionadas con el género. De acuerdo a la teoría de los recursos de poder, se sostiene que el creciente poder político de los actores que representan a los intereses de los sectores marginalizados de las clases populares, tales como, las organizaciones de trabajadores desocupados, sectores progresistas del movimiento sindical y los partidos de izquierda, creó las condiciones en las que fue posible un cambio hacia políticas sociales más redistributivas. El poder político cada vez mayor de estos actores no solo dependía de su creciente fuerza organizativa, sino también de la decadencia del pensamiento neoliberal ${ }^{3} y$ del descrédito de sectores de la élite vinculados a este pensamiento, que se da en Argentina hacia el nuevo centenario ${ }^{4}$.

Sin embargo, los cambios en la distribución de los recursos de poder no se traducen en cambios en las políticas de una manera mecánica. Para poder influir en el proceso de formulación de políticas, los actores habitualmente necesitan formar alianzas con otras fuerzas. Por su parte, este proceso de conformación de alianzas, influye en la forma en que una cierta distribución de recursos

3 De acuerdo con Peck y Tickell $(2002,394)$ se define "neoliberalismo" como una teoría económica y social que se "basa en una naturalización generalizada de la lógica del mercado", que prioriza el crecimiento económico ante cuestiones sociales y que postula un antagonismo entre políticas redistributivas y crecimiento económico.

Datos del World Value Survey muestran que las lógicas neoliberales dominaban la opinión pública durante los inicios de la década de los 90, pero que hacia finales de esa década una mayoría se pronunciaba en contra de estas a favor de un Estado más intervencionista y redistributivo. de poder se ve reflejada en políticas estatales. Por lo tanto, la teoría de los recursos de poder será combinada con enfoques teóricos que consideran el impacto de la formación de alianzas en la política redistributiva.

Uno de los enfoques más influyentes de este tipo emerge del análisis de Collier y Collier (2002) respecto de la incorporación política de los movimientos obreros en América Latina durante la primera mitad del siglo xx, la cual estaba relacionada con cambios significativos en las políticas distributivas. Sobre la base de este marco analítico, Collier y Handlin (2009) han sugerido recientemente que América Latina está viviendo otro momento crítico, en el curso del cual está teniendo lugar una importante transformación de la relación entre los Estados y los sectores marginalizados de las clases populares.

Para analizar más concretamente de qué manera un momento crítico define cambios en las políticas distributivas, el concepto de la "recomposición de la alianza gobernante" de Guillermo O’Donnell (1977) resulta muy útil. O'Donnell define alianza gobernante como la coalición de fuerzas "que impone, a traves del sistema institucional del Estado, políticas conformes a las orientaciones $y$ demandas de sus componentes" (1977, 551). Si cambia la composición de la alianza gobernante, es esperable que se dé un giro en las políticas estatales, ya que con ella cambian las orientaciones $y$ demandas que conviven dentro de la alianza y que requieren ser integradas a la agenda de políticas públicas. Por la gran capacidad que tienen las políticas sociales para influir en las condiciones de vida de los sectores marginalizados de las clases populares, se puede asumir que una integración de estos sectores a la alianza gobernante resulta en esfuerzos para ampliar la protección social para estos sectores, ya sea por presiones $y$ demandas articuladas por actores populares dentro de la alianza, o por la necesidad del conjunto de la alianza de mantener estos sectores dentro de la misma y de movilizar sus votos $y$ adhesiones en actos políticos.

Finalmente, cabe mencionar que la formación de alianzas gobernantes está estrechamente vinculada con la distribución de recursos 
de poder, ya que esta última determina qué tipos de alianzas son viables en un momento histórico dado y qué fuerzas son las que más pesan dentro de la alianza que eventualmente se conforme (O'Donnell 1977).

Dos estrategias metodológicas serán aplicadas con el fin de analizar empíricamente estos argumentos teóricos: la reconstrucción de procesos políticos (process tracing) y la comparación longitudinal de dos períodos de tiempo. La carga probatoria más importante para el argumento deriva de la reconstrucción de los procesos políticos que condujeron a la expansión de la política social. A través de la reconstrucción cronológica de la serie de eventos es posible visibilizar los mecanismos causales que vinculan la incorporación de los sectores marginalizados de las clases populares con la expansión de la protección social para estos grupos (Bennett 2010 y Collier 2011). La comparación longitudinal del período que precede $y$ del período en el que se expanden las políticas sociales se asemeja al tipo ideal ${ }^{5}$ de un "diseño de los casos más similares" (Przeworski y Teune 1970), lo cual provee una herramienta adicional para identificar aquellos factores que distinguen estos períodos en términos políticos y que por ende, podrían haber causado el cambio de rumbo en la política social.

\section{LAS TRANSFORMACIONES SOCIALES, ECONÓMICAS Y POLÍTICAS HACIA FINALES DEL SIGLO XX}

La implementación de las reformas neoliberales tras el golpe de Estado perpetrado por un grupo de líderes militares de derecha

5 Resulta importante hacer hincapié en que los diseños "de los casos más similares" y "de los casos más disímiles" son tipos ideales que en los análisis reales nunca pueden lograrse plenamente (Collier y Collier 2002, 15). Por lo tanto, se toman como punto de referencia y se trata de realizar un acercamiento a estos tanto como sea posible, mientras que también se reconocen los límites de estas estrategias comparativas. A través de la combinación de estas estratégias comparativas con la reconstrucción y el análisis de los procesos políticos se logra compensar las debilidades de una estrategia metodológica con la otra. en 1976, tuvo consecuencias devastadoras para las clases populares argentinas. La dictadura reprimió a los sindicatos, redujo drásticamente las barreras a las importaciones, desreguló el mercado financiero interno y liberalizó las transacciones financieras. El conjunto de estas medidas causó un fuerte aumento de la deuda externa, una drástica caída del salario real y una fuerte disminución de los puestos de trabajo formales en la industria (Beccaria, Groisman y Maurizio 2009). Además, estos procesos se vieron potenciados por las crisis de deuda que siguieron durante los años 80 . De esta manera, el nivel de pobreza escaló de alrededor del 5\% de la población en 1974, a casi el 40\% en el pico de la crisis hiperinflacionaria en 1989 (Beccaria 2007; Gasparini y Cruces 2010).

En ese mismo año, Carlos Saúl Menem del Partido Justicialista (Рı) ganó las elecciones presidenciales con una plataforma política basada en promesas de aumentos salariales y oposición al ajuste neoliberal. Sin embargo, inmediatamente después de asumir el cargo, Menem realizó un giro de 180 grados y adoptó una agenda de reestructuración neoliberal, incluyendo privatizaciones, liberalización del comercio internacional y desregulación del mercado interno (McGuire 1997). En materia de seguridad social, el gobierno apostó a la privatización del sistema previsional, a reglas más restrictivas para poder jubilarse y a la desregulación de los seguros de salud (Alonso 2000). Para controlar la inflación introdujo una nueva moneda y fijó la tasa de cambio en el uno-a-uno con el dólar estadounidense (McGuire 1997). En el corto plazo, la medida tuvo efectos positivos; puso fin a la hiperinflación y de esta forma permitió una recuperación parcial de los salarios reales y una reducción significativa de la desigualdad y la pobreza (Beccaria et ál. 2009). Estos éxitos contribuyeron a una amplia aprobación pública de las recetas neoliberales durante los primeros años de los años 90 (World Value Survey 2014). Sin embargo, en el mediano y largo plazo, estas políticas dieron lugar a una fuerte sobrevaloración del peso argentino que encareció la producción industrial local. Como consecuencia, se dio un profundo proceso de 
desindustrialización y deterioro socioeconómico (Beccaria et ál. 2009; Gasparini y Cruces 2010). La tasa de desempleo aumentó de 6,3\% en 1990 al 18,4\% en 1995 y en el trascurso de los años, la pobreza, la desigualdad y la informalidad llegaron a afectar a una parte cada vez mayor de la población (Instituto Nacional de Estadística y Censos de la República de Argentina-InDEc 2014).

En este contexto, la participación organizada de las clases populares en la política experimentó importantes transformaciones. La desindustrialización, el aumento de la informalidad y el desempleo debilitaron fuertemente al movimiento sindical (McGuire 1997). Además, el ajuste neoliberal generó una fuerte oposición de algunos sectores sindicales que finalmente llevaron a la conformación de la confederación sindical alternativa, la Central de Trabajadores de la Argentina (ста) (McGuire 1997). En contraste con la tradicional Confederación General del Trabajo (сGт), la ста permitió y fomentó la afiliación directa de trabajadores desempleados, informales $y$ jubilados (Fuentes, entrevista por Bossert, 21 de febrero de 2014; Larisgoitia, entrevista por Bossert, 9 de enero de 2014; Lozano, entrevista por Bossert, 28 de marzo de 2014). La defensa de los intereses de estos grupos económicamente marginalizados era considerada un interés del conjunto de la clase trabajadora entendida en un sentido amplio (Lozano, entrevista por Bossert, 28 de marzo de 2014). Por lo tanto, a lo largo de la década de los años 90 , la ста buscó activamente la cooperación con movimientos sociales organizados en barrios marginales (D’Elía, entrevista por Bossert, 19 de marzo de 2014 y Lozano, entrevista por Bossert, 28 de marzo de 2014).

En el corto y mediano plazo, el gobierno de Menem logró compensar la disminución del apoyo del movimiento sindical ${ }^{6}$ con la ampliación de su base electoral hacia la derecha del espectro político y el fortalecimiento de las

$6 \quad$ A pesar de la importante disminución del apoyo sindical, hay que reconocer que el gobierno de Menem logró conservar el apoyo y la disciplina de sectores más conservadores del sindicalismo. redes clientelistas ${ }^{7}$ de su partido en los crecientes barrios marginalizados (De Riz 1996; Levitsky 2003). Para las elecciones del 1995, el pJ conformó una alianza con el partido de derecha UceDé y ganó con amplio margen.

Sin embargo, durante la segunda mitad de la década de los 90 , esta estrategia encontró sus límites. La crisis económica de aquel entonces y el drástico deterioro de los indicadores socio-económicos fueron acompañados por un aumento significativo de la conflictividad social y un creciente rechazo público de la lógica neoliberal que el gobierno utilizó para justificar sus políticas (Svampa y Pereyra 2003; World Value Survey 2014). Además, movimientos sociales cada vez más fuertes, como la Federación de Tierra y Vivienda (FTV) y la Corriente Clasista y Combativa (ccc), comenzaron a competir con las estructuras locales del po por el liderazgo político en los barrios marginalizados. A través del bloqueo de carreteras importantes, desarrollaron la capacidad de hacer escuchar sus demandas a nivel nacional (Ardura, entrevista por Bossert, 13 de marzo de 2014; D'Elía, entrevista por Bossert, 19 de marzo de 2014). En este contexto, el gobierno introdujo un programa de empleo denominado Plan Trabajar y recibió a representantes de distintos movimientos sociales para negociar la distribución de una parte de los beneficios del programa entre los miembros de estos movimientos (Svampa y Pereyra 2003). Sin embargo, el programa nunca superó un promedio anual de 103000 beneficiarios (Golbert 2004), lo cual significa que cubría solamente una parte mínima de la población desempleada y necesitada ${ }^{8}$.

Hacia finales de 1998, la economía argentina entró nuevamente en recesión y en el año siguiente el ps perdió las elecciones presidenciales contra una alianza entre la "Unión Cívica Radical” (UCR) y el partido de centroizquierda "Frente País Solidario" (frepaso). Sin embargo, a pesar de poseer una plataforma

$7 \quad$ Se conceptualiza clientelismo político como "una relación basada en la subordinación política a cambio de recompensas materiales" (Fox 1994, 153).

8 En el mismo año hubo 1375000 personas desempleadas (INDEC 2014). 
electoral de centro-izquierda, el nuevo gobierno siguió con las políticas neoliberales de su predecesor. Los intentos de realizar recortes en el Plan Trabajar y la renuencia del gobierno a aceptar los movimientos sociales como socios legítimos de negociación condujeron a la multiplicación e intensificación de los cortes de ruta (Golbert 2004; Neffa et ál. 2008). A finales de octubre del 2000, los movimientos sociales bloquearon durante aproximadamente una semana la importante "Ruta 3" en el distrito de La Matanza en las afueras de Buenos Aires (Merklen 2005; Svampa y Pereyra 2003).

Al mismo tiempo, la ста inició el "Frente Nacional contra la Pobreza" (frenapo), que exigió acceso universal a la protección social, incluyendo un esquema de protección por desempleo no contributivo, la universalización de las asignaciones por hijo y una pensión mínima no contributiva. El FRENAPo fue apoyado por una amplia gama de organizaciones, incluyendo la FTV y la ccc (Lozano, entrevista por Bossert, 28 de marzo de 2014). En un referéndum informal durante diciembre de 2001, la propuesta recibió en pocos días más de 1,7 millones de firmas ( $\mathrm{La}$ Nación 2001b).

LA RECOMPOSICIÓN DE LA ALIANZA GUBERNAMENTAL Y LA EXPANSIÓN DE LA PROTECCIÓN SOCIAL HACIA LOS SECTORES MARGINALIZADOS

\section{EL GOBIERNO DE EDUARDO DUHALDE}

A principios del nuevo siglo, los sectores marginalizados de las clases populares habían alcanzado un poder político sin precedentes. Por un lado, los movimientos sociales habían crecido rápidamente $y$ forjado alianzas entre ellos. De acuerdo a entrevistas con líderes de la ccc (Ardura, entrevista por Bossert, 13 de marzo de 2014 ) y la FTv (D'Elía entrevista por Bossert, 19 de marzo de 2014), el número de miembros de estas organizaciones aumentó exponencialmente entre 2001 y 2002. La ccc creció en este lapso de 12000 a más de 50000 miembros. La fTV incluso llegó a tener 100000 miembros ${ }^{9}$. Se estima que la membresía de todos los movimientos sociales de este tipo alcanzó las 300000 personas (Alcañiz y Scheier 2007), y su capacidad de movilización y apelación incluso superó estos números. Por otra parte, estos movimientos sociales recibieron el apoyo de otras fuerzas sociales, tales como, la СТА, organizaciones de derechos humanos $y$ de los sectores progresistas de la Iglesia Católica. La movilización efectiva de estos recursos de poder desde finales de los 90 convirtió a estos movimientos en protagonistas de la resistencia contra las políticas neoliberales, las cuales ya habían perdido el apoyo público que habían disfrutado a principios de los 90 (Palomino 2005; World Value Survey 2014).

Además, la importancia de estos actores se vio, reforzada por la abrupta profundización de la crisis económica y social en el año 2001. En el contexto de una aceleración de la fuga de capitales, el gobierno restringió fuertemente la disponibilidad de dinero en efectivo de cuentas bancarias. Esta medida, a su vez, dio lugar a una ola de protestas por parte de las clases medias, que complicaron aún más la difícil situación política para el gobierno. El 19 de diciembre de 2001, los manifestantes de clase media $y$ de los movimientos sociales se unieron en la Plaza de Mayo frente a la casa de gobierno y exigieron la renuncia del presidente De la Rúa. El gobierno respondió con la declaración del Estado de sitio y reprimió fuertemente las protestas. Después de varios días de conflictos férreos, que arrojaron 26 muertes, De la Rúa finalmente renunció (Golbert 2004).

$\mathrm{El}$ FRENAPO reaccionó rápidamente y reiteró su propuesta de un programa de protección social universal poco antes de la designación de su sucesor (La Nación 2001b). En respuesta a la fuerza de las demandas sociales, el presidente interino Rodríguez Saá anunció en su discurso de presentación, la creación de un millón de puestos de trabajo e inmediatamente invitó a representantes de los movimientos

$9 \quad$ Estas cifras coinciden con las estimaciones del diario Perfil (30 de marzo 2007), que estima que la FTV y la CCC en conjunto alcanzaron una membresía de alrededor de 150000 durante el pico de la crisis de 2001-2002. 
sociales para negociar la distribución de cupos en el programa de trabajo (Ardura, entrevista por Bossert, 13 de marzo de 2014; Berra, entrevista por Bossert, 10 de marzo de 2014). Sin embargo, debido a la falta de apoyo de su propio partido (PJ) renunció después de solamente una semana en ejercicio del cargo (Golbert 2004).

Cuando Eduardo Duhalde, del mismo partido, asumió la presidencia, los movimientos piqueteros volvieron inmediatamente a las calles para exigir que se cumpla la promesa de un millón de puestos de trabajo. Líderes del FRENAPO reiteraron su demanda de un seguro de desempleo universal (La Nación 2002a y 2002b; Ardura, entrevista por Bossert, 13 de marzo de 2014; D’Elía, entrevista por Bossert, 19 de marzo de 2014). Consciente de la vulnerabilidad de su gobierno y de los recursos de poder de los movimientos sociales y del frenAPo, Duhalde anuncia la implementación de un esquema de protección al desempleo no contributivo llamado "Programa de Jefes y Jefas de Hogar Desocupados" (РлјнD) (Golbert 2004). Para legitimar su política invita a estas organizaciones ${ }^{10}$ a participar en una mesa de diálogo multisectorial (Neffa et ál. 2008; Ardura, entrevista por Bossert, 13 de marzo de 2014; D'Elía, entrevista por Bossert, 19 de marzo de 2014). Las dos centrales sindicales, сGт $y$ стА, diversas asociaciones de empleadores, los partidos políticos y representantes de la Iglesia Católica y otras religiones participaron en la mesa (López, entrevista por Bossert, 26 de marzo de 2014).

Una de las políticas que se discutieron en este espacio fue la propuesta del gobierno de implementar un esquema de protección al desempleo no contributivo. La propuesta original del gobierno restringió la cantidad de beneficiarios a 500000 y la duración del beneficio a solamente 3 meses (Golbert 2004; Decreto 165/2002). Estas restricciones fueron rechazadas por la стA, la ссC $y$ la FTV que a su vez, aprovecharon su participación en la mesa para demandar que se introdujeran políticas sociales más amplias que cubran a todos los necesitados

10 Además de la invitación a la mesa de diálogo, Duhalde se reunió con Luis D'Elía de la FTV y Chiquito Ardura de la ccc, el 17 de enero de 2002.
(Ardura, entrevista por Bossert, 13 de marzo de 2014; D'Elía, entrevista por Bossert, 19 de marzo de 2014; Lozano, entrevista por Bossert, 28 de marzo de 2014). Como resultado de las negociaciones se decidió levantar las restricciones, lo cual permitió una expansión sin precedentes de la cobertura (Neffa et ál. 2008). En 2003, el programa alcanzó 2130000 hogares, con lo cual su cobertura fue cuatro veces mayor que lo originalmente planeado por el gobierno (Golbert 2004).

El proceso de introducción de este programa permite observar cómo la transformación de la distribución de los recursos de poder y la consiguiente redefinición de la relación entre el gobierno nacional y los sectores marginalizados de las clases populares fueron decisivos para el cambio de la política social. Mientras que el gobierno del presidente De la Rúa se mostró reacio a recibir a los movimientos sociales como interlocutores en las negociaciones y respondió con represión a la protesta, tanto Rodríguez Saá como Duhalde reconocieron la necesidad de recibir a estos actores y negociar los términos de la política social con ellos ${ }^{11}$.

Sin embargo, la redefinición de la relación entre los sectores de bajos ingresos y el gobierno no constituyó un objetivo genuinamente buscado por el presidente Duhalde. Más bien fue una respuesta a la percibida necesidad política de hacer concesiones en un momento de profunda crisis. Tan pronto como la situación política parecía estabilizarse, el gobierno retornó a una postura más represiva y dejó de aceptar nuevos solicitantes al рлынд. Por lo tanto, solo el $62 \%$ de los hogares que cumplieron con los requisitos de elegibilidad recibieron efectivamente beneficios a través del programa (Neffa et ál. 2008).

La represión de las protestas de los movimientos sociales culminó con el homicidio de dos jóvenes durante un corte de ruta en junio de 2002; cientos más fueron heridos

11 La disposición de Rodríguez Saá y Duhalde de recibir a los movimientos sociales también fue propiciada por la histórica relación de su partido el PJ con los sectores económicamente marginalizados (Levitsky 2003: 41). 
(Svampa 2008). Esta escalada de violencia resultó ser altamente impopular y contribuyó finalmente a la decisión de Duhalde de no postularse como candidato presidencial en las elecciones del 2003 (Norden 2011).

\section{LOS GOBIERNOS KIRCHNERISTAS}

Una transformación más sólida de la relación entre el gobierno y los sectores marginalizados de las clases populares emergió bajo el gobierno de Néstor Kirchner, que deliberadamente persiguió la integración de estos sectores en su base de apoyo. Como gobernador de Santa Cruz, Néstor Kirchner había sido uno de los partidarios del FRENAPO. Su campaña electoral como candidato peronista de centro-izquierda se basó en una retórica fuertemente anti-neoliberal, la cual fue bien recibida por algunos de los movimientos sociales más fuertes. Sin embargo, en comparación con otros candidatos, Kirchner era relativamente desconocido a nivel nacional. Por lo tanto, forjar alianzas políticas, sobre todo en la populosa provincia de Buenos Aires, era indispensable para que su candidatura tuviera éxito. Además de varios acuerdos con líderes políticos y sindicales, forjó una alianza con la FTv (D'Elía, entrevista por Bossert, 19 de marzo de 2014). Finalmente, Kirchner llegó a la presidencia con poco más del $20 \%$ de los votos, porque su oponente, el dos veces presidente Carlos Menem, cedió de participar en el ballotage en vista de encuestas de opinión pública desfavorables.

Tras asumir como jefe del Ejecutivo, Kirchner apuntó a la consolidación de la alianza con los sectores marginalizados de las clases populares, convirtiéndolos en una importante base de apoyo electoral y organizativo. Tan solo unos días después de su asunción, Kirchner recibió a líderes de la FTv y la ccc, las dos organizaciones más grandes de trabajadores desempleados en la provincia de Buenos Aires. Poco tiempo después, también invitó a los líderes de organizaciones más pequeñas, de las cuales algunas eran claramente opositoras. El gobierno estableció una comunicación fluida con aquellos movimientos sociales, que se consideraban potenciales aliados políticos. Durante el primer año de mandato hubo frecuentes reuniones entre representantes de alto rango de la presidencia $y$ líderes de los movimientos sociales. Kirchner mismo participó en esas reuniones en varias ocasiones, haciendo hincapié en que él estaba dispuesto a defender los intereses de los sectores populares, pero que para ello los movimientos sociales tendrían que apoyar a su gobierno (Boyanovsky 2010). Mientras Luis D'Elía (Fтv) declaró su apoyo desde el principio, los líderes de otros movimientos sociales, como la ccc, Barrios de Pie y Movimiento de Trabajadores Desocupados Evita (мтр Evita), fueron inicialmente más cautelosos (Boyanovsky 2010; D’Elía, entrevista por Bossert, 19 de marzo de 2014). Para ganar el apoyo de estas organizaciones y de los grupos sociales que representaban, el gobierno aplicó una estrategia basada en diferentes medidas, pero en la cual la implementación de políticas sociales focalizadas y universalistas desempeñó un papel crucial.

La distribución de los beneficios de los programas focalizados, tales como los programas de empleo y de vivienda, favoreció a aliados leales y sirvió para negociar apoyo político en diferentes niveles de la organización política. Por un lado, las unidades básicas del ps en los barrios marginales continuaron funcionando como redes clientelares. Al distribuir beneficios de los programas sociales, estas redes sirvieron para establecer vínculos directos con personas de bajos ingresos (Etchemendy y Garay 2011). En un nivel más agregado, la distribución selectiva de recursos a través de los programas de vivienda y de empleo constituyó un componente importante de las negociaciones con los movimientos sociales y líderes locales del pu (Etchemendy y Garay 2011; Svampa 2008; Ardura, entrevista por Bossert, 13 de marzo de 2014).

Inicialmente, esta estrategia parecía ser relativamente exitosa. Barrios de Pie y мтр Evita pronto se sumaron a la alianza gobernante, afirmando que iban a apoyar al gobierno siempre que representara los intereses de los sectores marginalizados de las clases populares (Boyanovsky 2010). La ccc también tomó una posición amigable con el gobierno durante el primer tiempo de la presidencia de Kirchner (Massetti 2006). En los años subsiguientes, se fundaron nuevos movimientos sociales "kirchneristas", como el movimiento Evita y el Frente 
Transversal. La colaboración con el gobierno era ventajosa para estos movimientos sociales y en particular para sus líderes. La distribución de los recursos de los programas asistenciales $y$ de inversión social estuvo fuertemente sesgada a favor de las organizaciones leales. En última instancia, esto aumentó tanto la capacidad de los grupos leales para atraer nuevos miembros como el control de sus líderes sobre sus bases por medio de la distribución interna de los beneficios (Golbert 2004; Merklen 2005; Svampa 2008 y Falcon, entrevista por Bossert, 15 de enero de 2014). Adicionalmente, varios líderes de movimientos sociales asumieron cargos en diferentes niveles del gobierno (Norden 2011; Svampa 2008; La Nación 2006 y Página 12 2010. A cambio, estas organizaciones movilizaron con frecuencia a sus miembros en apoyo del gobierno, tanto electoralmente como en la calles (Boyanovsky 2010 y Norden 2011).

Por el contrario, los movimientos sociales de oposición, sufrieron una creciente fragmentación y pérdida de membresía (Massetti 2006 y Svampa 2008). Esto fue, en parte, el resultado de la distribución desigual de los beneficios selectivos de los programas sociales focalizados, así como, las estrategias judiciales y discursivas del gobierno para contrarrestar las organizaciones más radicales. Pero también era un reflejo de la gran popularidad del gobierno entre los sectores marginalizados de las clases populares, como resultado de sus políticas sociales, económicas y simbólicas. Sin embargo, los diversos movimientos sociales opositores mantuvieron cierta capacidad de movilización, al exigir acceso a los programas sociales y criticar el clientelismo en la selección de los beneficiarios (Ardura, entrevista por Bossert, 13 de marzo de 2014), las protestas de los movimientos sociales opositores contribuyeron a convertir el clientelismo en un tema prácticamente omnipresente en los medios de comunicación ${ }^{12}$.

12 Un análisis de la edición digital del diario El Clarín $y$ de las ediciones impresas y en línea del diario $L a$ Nación muestra que en 1997 se publicaron solo 6 artículos en El Clarín y 26 en La Nación que mencionaban la palabra "clientelismo". Con el aumento de los conflictos en torno a la distribución de los beneficios de la asistencia social y el fortalecimiento
Además, las frecuentes disputas sobre la distribución de beneficios entre diferentes movimientos sociales y políticos locales crearon tensiones permanentes dentro de la alianza gobernante. En conjunto con la heterogeneidad política de los movimientos sociales en la alianza, dichas tensiones contribuyeron a las decisiones de varias organizaciones importantes, como la ccc y Barrios de Pie, de retornar a estrategias más confrontativas (Norden 2011; Svampa 2008; La Nación 2012 y Ardura, entrevista por Bossert, 13 de marzo de 2014). La FTV se mantuvo leal al gobierno, pero hacia el 2009, la distribución desigual de los beneficios de un nuevo programa de empleo denominado "Ingreso social con Trabajo" entre los diferentes movimientos sociales provocó una grave crisis en la relación de la fTv con el gobierno (D'Elía, entrevista por Bossert, 19 de marzo de 2014). Por lo tanto, aunque los programas sociales focalizados desempeñaron un papel importante para ganar el apoyo de algunos movimientos sociales, la fortaleza de los movimientos también impuso límites a esta estrategia.

A partir de 2005, el gobierno puso más énfasis en la implementación de políticas sociales universalistas basadas en derechos (Midalgia 2012). Aunque estas políticas permitieron un nivel de control mucho menor sobre los beneficiarios, también resultaron mucho menos conflictivos y más aceptados incluso por fuerzas opositoras. A través del "Plan de Inclusión Previsional" se garantizó el derecho a una jubilación a las personas en edad jubilatoria que no hayan podido cumplir con el requisito de años de aportes. Esto dio lugar a un enorme aumento de la cobertura del sistema previsional entre los sectores marginalizados de las clases populares (Arza 2009). La cobertura de las personas mayores que viven en hogares del quintil de ingresos más bajos aumentó de $42 \%$ en 2004

de los movimientos de trabajadores desempleados durante los años siguientes, el número de artículos que mencionaban la palabra clientelismo aumentó rápidamente hasta llegar a 87 y 209, respectivamente, en 2003. A partir de 2003, el número de artículos que mencionaban la palabra ha fluctuado en un alto nivel en los dos periódicos, alcanzando picos en años electorales. 
a $85 \%$ en 2010 , y para el segundo quintil de ingresos más bajos el aumento fue del $66 \%$ al 95\%, respectivamente (Alonso y Di Costa 2012). A su vez, el gobierno aumentó regularmente las jubilaciones mínimas, con lo cual la distribución de los recursos dentro del grupo de los jubilados se hizo cada vez más favorable para los sectores de bajos ingresos ${ }^{13}$.

En 2007, Cristina Fernández de Kirchner de la alianza kirchnerista ganó las elecciones presidenciales con un amplio margen. Sin embargo, dos años más tarde, en medio de la crisis financiera mundial y después de un conflicto políticamente costoso con los productores agrícolas, la alianza kirchnerista sufrió graves pérdidas en las elecciones legislativas. En este contexto, el gobierno emprendió un nuevo esfuerzo para ampliar la protección social para los sectores marginalizados de las clases populares. En agosto de 2009, introdujo un programa social focalizado llamado "Ingreso Social con Trabajo" que proporcionó aproximadamente 100000 puestos de trabajo en cooperativas con un salario mensual de 1200 pesos (314 dólares) (Vales 2009). Claramente, esta política respondió tanto a la meta de paliar los efectos sociales de la crisis, como a la necesidad política de reforzar el apoyo de los sectores de bajos ingresos y sus movimientos sociales. El programa se inspiró en propuestas realizadas por algunos movimientos sociales aliados al gobierno, en particular del Movimiento Evita (Natalucci 2012).

Paralelamente a la implementación, dicho movimiento lanzó una campaña masiva de afiches en apoyo a Néstor Kirchner, en aquel entonces previsto como candidato a presidente del Kirchnerismo, en 2011. Lo que el gobierno no había esperado era que, poco después, la desigual distribución de los recursos del programa

13 Mientras que la pensión mínima constituía solo el 41,8\% de la pensión media en 2001, alcanzó el $77,8 \%$ de la misma en 2010 (cálculos propios sobre la base de MTEYSS 2012). El financiamiento para la expansión de la cobertura y el aumento de las jubilaciones mínimas provino principalmente de tres fuentes: el aumento del empleo formal, la reducción relativa de las jubilaciones más altas y la estatización de los fondos privados de pensiones (MTEYSS 2012). dio origen a repetidas y masivas oleadas de bloqueos de carreteras por parte de los movimientos sociales opositores, así como, fuertes críticas de los partidos de la oposición (Guimenez y Hopp 2011). Estas protestas, a su vez, dieron lugar a que los medios de comunicación emitieran noticias perjudiciales para el gobierno ${ }^{14}$, que no solo denunciaron las prácticas clientelistas, sino también responsabilizaron al gobierno por los masivos bloqueos de carreteras, que agravaron aún más la complicada situación de tránsito en la aglomeración de Buenos Aires. Además, la distribución selectiva de los beneficios del programa llevó a tensiones considerables entre los movimientos sociales dentro de la alianza gobernante. Los dos movimientos sociales más favorecidos en el desembolso de beneficios fueron el Movimiento Evita, que pudo registrar entre 15000 (La Nación 2010a) y 30000 (La Nación 2010b) miembros en el programa, y el Frente Transversal, que pudo de registrar más de 5000 (La Nación 2010a) miembros. Por el contrario, la FTV recibió considerablemente menos beneficios y por lo tanto, comenzó a expresar públicamente su descontento con el gobierno (D’Elía, entrevista por Bossert, 19 de marzo de 2014). En resumen, el programa Ingreso Social con Trabajo arrojó resultados dudosos con respecto a la movilización del apoyo de los sectores marginalizados de las clases populares.

Al mismo tiempo, la demanda de medidas de protección social más universalistas, especialmente para los niños, se hizo cada vez más fuerte, tanto dentro de la oposición como dentro de la alianza política del gobierno (Linares, entrevista por Bossert, 25 de febrero de 2014 y Lozano, entrevista por Bossert, 28 de marzo de 2014). Entre 2008 y 2009, cinco proyectos de ley fueron presentados por diferentes legisladores que propusieron una extensión del sistema de asignaciones a los hijos de trabajadores informales y desempleados. Uno de ellos fue

\footnotetext{
14 Las negociaciones y los conflictos acerca del programa han sido documentados en cientos de artículos periodísticos. Dado que no es posible hacer referencia a todos los artículos correspondientes, se mencionan tres ejemplos: La Nación, 30 de diciembre, 2009; Página 12, 4 de noviembre, 2009; El Clarín, 9 de noviembre, 2009.
} 
incluso presentado por un legislador de la alianza kirchnerista. En octubre de 2009, también la Iglesia Católica propuso públicamente una medida similar (Hintze y Costa 2011). En este contexto, el gobierno decidió apropiarse de la propuesta de una política universalista dirigida hacia los niños. A finales de octubre de 2009, solo tres meses después de la introducción del programa Ingreso Social con Trabajo, se introdujo por decreto la Asignación Universal por Hijo (аUн). Con la aur se extendió el sistema de asignaciones por hijo a los trabajadores informales y desempleados, proporcionando un pago mensual de 180 pesos por niño (\$47us). Asimismo, sustituyó varios programas selectivos y cubrió alrededor de 3,5 millones de niños en hogares de bajos ingresos. Aunque el beneficio está relacionado con cierto condicionamiento, constituye un derecho para todas las familias que cumplan con los requisitos legales (Gasparini y Cruces 2010). En contraste con el programa Ingreso Social con Trabajo, la AuH recibió amplio apoyo público y se convirtió en uno de los logros más explotados en los discursos y material de campaña en las elecciones presidenciales del 2011.

En resumen, hacia finales de la primera década del nuevo milenio, Argentina había aumentado considerablemente el gasto social destinado a los sectores económicamente marginalizados y el gobierno había consolidado el apoyo organizativo y electoral de dichos sectores.

\section{CONCLUSIONES}

La comparación de los dos períodos, uno anterior y uno durante la fase de expansión de la política social argentina, muestra que un cambio significativo en la dirección de la política social tuvo lugar desde el momento en que se comenzó a cristalizar una recomposición de la alianza gobernante. En la nueva alianza, los sectores marginalizados de las clases populares tuvieron un peso político mayor $y$ algunos de sus movimientos sociales accedieron a nuevos canales de participación en la definición de políticas.

La reconstrucción y el análisis del proceso político subyacente revelan cómo las transformaciones de los contextos económico, social $y$ discursivo, contribuyeron a la recomposición de la alianza gobernante y qué mecanismos causales concretos relacionaron esta recomposición con los profundos cambios que se fueron implementando en el campo de la política social.

Las crisis económicas y el ajuste neoliberal de fines del siglo xx condujeron a un proceso de desindustrialización y un aumento del desempleo, de la informalidad y de la desigualdad. En el marco de estas transformaciones, la clase trabajadora formal y los sindicatos tradicionales se debilitaron significativamente, mientras que las filas de los sectores marginalizados de las clases populares no dejaron de crecer. De esta manera, las organizaciones sindicales tradicionales perdieron recursos de poder, mientras que los movimientos sociales que representan a los sectores marginalizados cobraron fuerza y lograron un importante nivel de prominencia en la resistencia contra las políticas neoliberales cada vez más impopulares. Al mismo tiempo, el descrédito de las políticas neoliberales entre la mayoría de los argentinos significó que propuestas de políticas redistributivas volvieran a ser más viables en términos electorales. En conjunto estos desarrollos crearon condiciones favorables para una recomposición de la alianza gobernante, que reflejó el incremento del peso político de los sectores económicamente marginalizados $y$ sus expresiones organizadas. Tal proceso de recomposición se inició con la crisis del 20012002, y se cristalizó de forma más clara con la conformación y consolidación de la alianza gobernante kirchnerista, que tiene como una de sus principales bases de apoyo a los sectores marginalizados de clases populares $y$ varios de sus movimientos sociales.

Mediante la reconstrucción de los procesos políticos subyacentes, el presente estudio pudo identificar dos tipos de mecanismos causales que relacionaron esta recomposición de la alianza con la expansión de las políticas sociales. El primero de estos mecanismos fue que la integración de varios de los movimientos sociales les permitió participar, aunque de manera restringida, en la definición de políticas sociales mediante propuestas propias $y$ críticas a los planes de la cúpula de la alianza. 
En algunas ocasiones estos procesos de negociación dentro de la alianza involucraron importantes tensiones. Dados los recursos de poder de estos movimientos sociales y sus capacidades de movilización, su peso político dentro de la alianza fue significativo.

El segundo mecanismo que se pudo observar a lo largo del período analizado fue que la cúpula de la alianza gobernante utilizó la expansión de la política social no solamente como herramienta para alcanzar una redistribución de ingresos de acuerdo con sus convicciones ideológicas, sino también para consolidar y reforzar su base de apoyo entre los sectores marginalizados de las clases populares. Para este fin, políticos y funcionarios de la alianza se refirieron deliberadamente a las políticas sociales en sus discursos y material de campaña, con el fin de demostrar su compromiso con los intereses de estos sectores. En algunos casos, la movilización de apoyos mediante políticas sociales focalizadas también involucró prácticas clientelistas.

\section{BIBLIOGRAFÍA}

LIBROS

Alonso, Guillermo. 2000. Política y seguridad social en la Argentina de los ,90. Buenos Aires: Miño y Dávila Editores.

Beccaria, Luis. 2007. "Pobreza". Población y bienestar en la Argentina del primero al segundo centenario. Susana Torrado (ed.). Buenos Aires: Edhasa.

Beccaria, Luis; Fernando Groisman y Roxana Maurizio. 2009. "Notas sobre la Evolución Macroeconómica y del Mercado de Trabajo en la Argentina 1975-2007". Argentina Desigual. Luis Beccaria y Fernando Groisman (eds.). Buenos Aires, Argentina: Prometeo.

Bennett, Andrew. 2010. "Process Tracing and Causal Inference". Rethinking social inquiry: diverse tools, shared standards. Henry E. Brady y David Collier (eds.). Lanham: Rowman and Littlefield.

Boyanovsky Bazán, Christian. 2010. El Aluvión. Del Piquete al Gobierno. Los Movimientos Piqueteros $y$ el Kirchnerismo. Buenos Aires: Sudamericana.

Collier, Ruth Berins y David Collier. 2002. Shaping the Political Arena: Critical Junctures, the Labor Movement, and Regime Dynamics in Latin America. Princeton: Princeton University Press.

Collier, Ruth Berins y Samuel Handlin. 2009. Reorganizing Popular Politics: Participation and the New Interest Regime in Latin America. University Park: Pennsylvania State University Press.

De Ferranti, David. 2004. Inequality in Latin America: Breaking with History? Washington: World Bank.

De Riz, Liliana. 1996. "Menem-Zweiter Akt. Die Wiederwahl". En Argentinien. Politik, Wirtschaft, Kultur und Außenbeziehungen, editado por Detlef Nolte y Nikolaus Werz. Frankfurt am Main: Vervuert.

Esping-Andersen, Gøsta. 1990. The Three Worlds of Welfare Capitalism. Princeton: Princeton University Press.

Etchemendy, Sebastián y Candelaria Garay. 2011. "Argentina: Left Populism in Comparative Perspective, 2003-2009”. En The Resurgence of the Latin American Left, editado por Steven Levitsky y Kenneth Roberts. Baltimore: Johns Hopkins University Press.

Fiszbein, Ariel. 2005. Beyond Truncated Welfare Slates: Quo Vadis Latin America? Washington: World Bank.

Haggard, Stephan y Robert R. Kaufman. 2008. Development, Democracy and Welfare States: Latin America, East Asia, and Eastern Europe. Princeton: Princeton University Press.

Hintze, Susana y María Ignacia Costa. 2011. "La reforma de las Asignaciones Familiares 2009. Aproximación al proceso político de la transformación de la protección". En Protecciones y Desprotecciones. La Seguridad Social en la Argentina 19902010. Claudia Danani y Susana Hintze (eds.). Los Polvorines: Universidad Nacional de General Sarmiento. 
Huber, Evelyne y John D. Stephens. 2001. Development and Crisis of the Welfare State: Parties and Policies in Global Markets. Chicago: University of Chicago Press.

Huber, Evelyne y John D. 2012 Stephens. Democracy and the Left: Social Policy and Inequality in Latin America. Chicago: University of Chicago Press.

Korpi, Walter. 1983. The Democratic Class Struggle. London: Routledge \& Paul.

Levitsky, Steven. 2003. Transforming LaborBased Parties in Latin America: Argentine Peronism in Comparative Perspective. Cambridge: Cambridge University Press.

Lustig, Nora; Luis F. Lopez-Calva y Eduardo Ortiz-Juarez. 2012. Declining Inequality in Latin America in the 2000s: The Cases of Argentina, Brazil, and Mexico. Washington: World Bank.

McGuire, James. 1997. Peronism without Perón: Unions, Parties, and Democracy in Argentina. Stanford: Stanford University Press.

Merklen, Denis. 2005. Pobres Ciudadanos. Las clases populares en la era democrática. Argentina, 1983-2003. Buenos Aires: Gorla.

Ministerio de Trabajo, Empleo y Seguridad Social (мтеуss). 2012. Boletín Estadístico de la Seguridad Social. Buenos Aires: MTEYSS.

Neffa, Julio César; Agustina Battistuzzi; Cecilia Del Bono, Adrián Des Champs, Alejandro Enrique y Ezequiel Grinberg. 2008. Desempleo, pobreza y politicas sociales. Fortalezas y debilidades del Plan Jefes y Jefas de Hogar Desocupados. Buenos Aires y Madrid: Miño y Dávila.

Palomino, Héctor. 2005. "Los Sindicatos y los Movimientos Sociales Emergentes del Colapso Neoliberal en Argentina”. En Sindicatos y Nuevos Movimientos Sociales en América Latina, compilado por Enrique De la Garza Toledo. Buenos Aires: Consejo Latinoamericano de Ciencias Sociales (clacso).

Przeworski, Adam y Henry Teune. 1970. The Logic of Comparative Social Inquiry.
New York: Wiley-Interscience.

Svampa, Maristella y Sebastián Pereyra. 2003. Entre la Ruta y el Barrio. Las Experiencias de las Organizaciones Piqueteras. Buenos Aires: Biblos.

\section{PUBLICACIONES PERIÓDICAS}

Alcañiz, Isabella, y Melissa Scheier. 2007. "New Social Movements with Old Party Politics. The mть Piqueteros and the Communist Party in Argentina”. Latin American Perspectives 2, n. ${ }^{\circ}$ 34: 157-171.

Aulicino, Eduardo. 2009. "Piqueteros vs. Intendentes: pelea por los planes sociales y por el aparato K". El Clarín, 9 de noviembre. Acceso el 10 de octubre de 2010. <http://edant.clarin.com/ diario/2009/11/09/elpais/p-02036752.htm

Barrientos, Armando. 2012. "Dilemas de las politicas sociales latinoamericanas. ¿Hacia una protección social fragmentada?". Nueva Sociedad 239: 65-78.

Becker, Ulrich, y Carmelo Mesa-Lago. 2013. "Re-Reformas de sistemas de pensiones privatizadas en el mundo: estudio comparativo de Argentina, Bolivia, Chile y Hungría”. Revista Trabajo 10, 7: 43-53.

Collier, David. 2011. "Understanding Process Tracing”. Political Science \& Politics 44, 4: 823-830.

Fox, Jonathan. 1994. "The difficult transition from clientelism to citizenship: lessons from Mexico". World Politics 46 (2). Princeton, EEUU: Princeton Institute for International and Regional Studies, 151-184.

Golbert, Laura. 2004. "¿Derecho a la inclusión o paz social? Plan Jefes y Jefas de Hogar Desocupados“. Serie de Políticas Sociales 84. Santiago de Chile: cEpal.

Hobson, Barbara y Marike Lindholm. 1997. "Collective Identities, Women's Power Resources, and the Making of Welfare States". Theory and Society 4, 26: 475-508.

La Nación. 2001a."D’Elía Convocó a una Nueva Asamblea de Piqueteros". La Nación. 16 de agosto. Acceso el 10 de junio de 2012. http://www.lanacion.com.ar/328101delia-convoco-a-una-nueva-asamblea-depiqueteros 
La Nación. 2001b. "El Frenapo presentará un Plan Económico y Social al Parlamento". La Nación. 22 de diciembre. Acceso el 12 de junio de 2012. http://www.lanacion. com.ar/361370-el-frenapo-presentara-unplan-economico-y-social-al-parlamento

La Nación. 2002a."El Frenapo le pidió a Duhalde un seguro de desempleo". La Nación. 14 de enero. Acceso el 17 de mayo de 2014. http://www.lanacion.com. ar/366644-el-frenapo-le-pidio-a-duhaldeun-seguro-de-desempleo

La Nación. 2002b. "Protesta de jubilados y desocupados en el centro porteño". La Nación. 15 de enero. Acceso el 17 de mayo de 2014. http://www.lanacion. com.ar/366854-protesta-de-jubilados-ydesocupados-en-el-centro-porteno

La Nación. 2006."Cincuenta piqueteros cambiaron la calle por los sillones del poder". La Nación. 11 de junio. Acceso el 10 de octubre de 2010. http://www. lanacion.com.ar/813651-cincuentapiqueteros-cambiaron-la-calle-por-lossillones-del-poder

La Nación. 2009.“Piqueteros Antikirchneristas provocaron complicaciones en el tránsito". La Nación. 30 de diciembre. Acceso el 14 de octubre de 2010. http:// www.lanacion.com.ar/1216854piqueteros-antikirchneristas-provocaroncomplicaciones-en-el-transito

La Nación. 2010a."Pérsico, el ganador en el reparto de planes". La Nación. 14 de febrero Acceso el 27 de octubre de 2010. http:// www.lanacion.com.ar/1233050-persico-elganador-en-el-reparto-de-planes

La Nación. 2010b. "Las Cooperativas como herramienta electoral". La Nación. 3 de septiembre. Acceso el 27 de octubre de 2010 En: http://www.lanacion.com. ar/1300755-las-cooperativas-comoherramienta-electoral

Martínez Franzoni, Juliana y Koen Voorend. 2009. "The Role of Distributional Coalitions in Welfare Regimes: Chile, Costa Rica and El Salvador". Social Policy \& Administration 4, 43: 364-381.
Massetti, Astor. 2006. "Piqueteros eran los de Antes': Sobre las Transformaciones en la Protesta Piquetera". Lavboratorio 19, 8: 29-36.

Midalgia, Carmen. 2012. "Un Balance Crítico de los Programas Sociales en América Latina. Entre el Liberalismo y el Retorno del Estado". Nueva Sociedad 239: 79-89.

Natalucci, Ana. 2012. "Políticas Sociales y Disputas Territoriales. El Caso del Programa Argentina Trabaja”. Revista de Perspectivas de Politicas Pública 3: 126-147.

Norden, Deborah. 2011. "The Policies and Politics of Control: Countering Argentina's Uniformed and Unemployed Protesters". Latin American Politics and Society 3, 53: 83-114.

O'Donnell, Guillermo. 1977. "Estado y alianzas en Argentina”. Desarrollo Económico xvı 64: 523-554.

Página 12. 2010. "Depetri a una Subsecretaría." Página 12. 17 de marzo. Acceso el 10 de octubre de 2013. http:// www.pagina 12 .com.ar/diario/ elpais/1-142179-2010-03-17.html

Peck, Jamie y Adam Tickell. 2002. "Neoliberalizing Space". Antipode 3, 34: 380-404.

Svampa, Maristella. 2008. "Argentina: una Cartografía de las Resistencias (20032008). Entre las luchas por la Inclusión y las discusiones sobre el Modelo de Desarrollo". Revista osal 24, 9: 17-49.

Vales, Laura. 2009. "Los Piqueteros Levantaron Campamento". Página 12. 4 de noviembre. Acceso el 10 de octubre de 2010. http://www.pagina12.com.ar/diario/ elpais/1-134667-2009-11-04.html

\section{TEXTOS ELECTRÓNICOS}

Perfil, versión en línea. 2007. Emilio Pérsico y Luis D'Elia. Los fieles guardianes de la causa K. 30 de marzo. Acceso el 21 de diciembre de 2010. http://www. diarioperfil.com.ar/edimp/0247/articulo. php?art $=6500 \&$ ed $=0247$ 
ENTREVISTAS

Ardura, Chiquito (Coordinador Nacional de la $\mathrm{ccc})$, entrevista por Bossert, el 13 de marzo de 2014.

Berra, Claudia (Área de políticas sociales en el Ministerio de Trabajo), entrevista por Bossert, el 10 de marzo de 2014.

D’Elía, Luis (Secretario general de la FTv), entrevista por Bossert, el 19 de marzo de 2014.

Eduardo Falcón, Martín (Secretario sindical del sindicato sutna $y$ ex-militante del Movimiento Evita), entrevista por Bossert, el 15 de enero de 2014.

Larisgoitia, Andrés (Director de la Secretaría de Relaciones Internacionales de la ста), entrevista por Bossert, el 9 de enero de 2014.

Linares, Virginia (Diputada nacional por el partido GEN), entrevista por Bossert, el 25 de febrero de 2014.

Lopez, José Ignacio (Participante en la organización de la mesa de diálogo como empleado del PNuD), entrevista por Bossert, el 26 de marzo de 2014.

Fuentes, Julio (Secretario general del sindicato AтE $y$ dirigente de la ста), entrevista por Bossert, el 21 de febrero de 2014.

Lozano, Claudio (Diputado nacional por el partido Unidad Popular y dirigente de la стА), entrevista por Bossert, el 28 de marzo de 2014.

OTROS

Alonso, Guillermo y Valeria Di Costa. 2012. "Más allá del Principio Contributivo. cambios y continuidades en la política social Argentina. 2003-2011". Annual Meeting of the Latin American Studies Association (Lasa). San Francisco, 23 al 26 de mayo.

Arza, Camila. 2009. "Back to the State: Pension Fund Nationalization in Argentina". UNRISD Conference. Geneva, 12 y 13 de noviembre.

Comisión Económica para América Latina $y$ el Caribe (CEPAL). 2013. Social Panorama of Latin America 2013. Santiago de Chile: cepal.

Comisión Económica para América Latina y el Caribe (cepal). 2014. Pactos para la igualdad: hacia un futuro sostenible. Santiago de Chile: cEpal.

Gasparini, Leonardo y Guillermo Cruces. 2010. "Las Asignaciones Universales por Hijo: Impacto, discusión y alternativas". Working Paper 102. La Plata: cedlas.

Guimenez, Sandra y Malena Hopp. 2011. "Programa Ingreso Social con Trabajo 'Argentina Trabaja': una mirada reflexiva desde el corazón de su implementación". IV Encuentro Internacional de Trabajo Social de la Universidad de Buenos Aires. Buenos Aires, Argentina. 12 y 13 de mayo.

Instituto Nacional de Estadística y Censos de la República de Argentina. Acceso el 24 de junio de 2014. http://www.indec. mecon.ar/

World Value Survey. Acceso el 24 de junio de 2014. http://www.worldvaluessurvey.org/ wvs.jsp

Fecha de ingreso: 16/04/2015 Fecha de aprobación: 23/07/2015 
